\title{
The Impact of Type Ia Supernova Ejecta on Binary Companions
}

\author{
P. M. Ricker*, K.-C. Pan* and R. E. Taam ${ }^{\dagger, * *}$ \\ ${ }^{*}$ Dept. of Astronomy, Univ. of Illinois, Urbana, IL 61801 \\ ${ }^{\dagger}$ Dept. of Physics and Astronomy, Northwestern Univ., Evanston, IL 60208 \\ **Academia Sinica Institute of Astronomy and Astrophysics, Taipei 10617, Taiwan
}

\begin{abstract}
We present adaptive mesh refinement (AMR) hydrodynamical simulations of the interaction between Type Ia supernovae and their companion stars within the context of the single-degenerate model. Results for 3D red-giant companions without binary evolution agree with previous 2D results by Marietta et al. We also consider evolved helium-star companions in 2D. For a range of helium-star masses and initial binary separations, we examine the mass unbound by the interaction and the kick velocity delivered to the companion star. We find that unbound mass versus separation obeys a power law with index between -3.1 and -4.0 , consistent with previous results for hydrogen-rich companions. Kick velocity also obeys a power-law relationship with binary separation, but the slope differs from those found for hydrogen-rich companions. Assuming accretion via Roche-lobe overflow, we find that the unbound helium mass is consistent with observational limits. Ablation (shock heating) appears to be more important in removing gas from helium-star companions than from hydrogen-rich ones, though stripping (momentum transfer) dominates in both cases.
\end{abstract}

Keywords: Type Ia supernovae; binary stars; hydrodynamics

PACS: $97.60 . \mathrm{Bw}, 97.80 . \mathrm{Kq}$

\section{INTRODUCTION}

Type Ia supernovae (SNe Ia), unlike core-collapse supernovae, are most likely purely a phenomenon of close binary systems. Their defining absence of hydrogen spectral lines and presence of silicon lines suggest an origin in compact stellar remnants, while the progenitor delay times inferred from their presence in early- and late-type galaxies and their absence from interarm regions require these remnants to be low-mass, ie. white dwarfs (WDs). The relative homogeneity of the SN Ia population suggests that most white dwarfs explode at a single mass, probably the Chandrasekhar mass, $1.44 M_{\odot}$ [1]. Because the distribution of white dwarfs peaks at $0.6 M_{\odot}$ [2], Chandrasekharmass models require that the white dwarf accrete from a binary companion.

Most work on progenitor models for SNe Ia has therefore focused on two general cases: the single-degenerate model, in which a white dwarf accretes matter from a normal stellar binary companion, then becomes unstable to explosive nuclear burning [1,3], and the double-degenerate model, in which two white dwarfs merge due to gravitationalwave emission $[4,5]$. Single-degenerate models must cope with the problem of the companion star's hydrogen and helium; observational limits on $\mathrm{H} / \mathrm{He}$ abundances in SNe Ia are very constraining [6, 7]. These models also have the difficulty that the mass accretion rate onto the white dwarf must be in a fairly narrow range above $10^{-7} M_{\odot} \mathrm{yr}^{-1}$ to avoid either nova outbursts or development of a white-dwarf wind that limits the accretion efficiency [3, 8, 9]. This makes it difficult to explain the observed low-redshift SN Ia rate of $3 \times 10^{-5} \mathrm{Mpc}^{-3} \mathrm{yr}^{-1}$ [10] using single-degenerate progenitors alone. The predicted and observed abundances of double-degenerate systems are sufficient to explain the observed rate $[11,12,13]$. However, such collisions may lead to production of an O-Ne-Mg white dwarf followed by accretion-induced collapse to a neutron star $[14,9,15,16]$. It is possible that multiple progenitor channels contribute. The observed redshift distribution of SNe Ia is consistent with at least two populations having different delay times measured from the zero-age main sequence [10].

We are examining the single-degenerate model to determine, among other things, whether the problem of $\mathrm{H} / \mathrm{He}$ in the companion envelope can be overcome. Single-degenerate progenitors can explode at the Chandrasekhar mass for main-sequence (MS), red-giant (RG), or helium-star companions. If the companion is a helium star, the WD may also rarely explode below the Chandrasekhar mass via explosive burning of an accreted helium layer that ignites interior carbon burning [17]. The Chandrasekhar-mass helium-star channel is regarded as a candidate for the short delay-time population of SNe Ia because this case requires a massive initial MS star [18, 19, 20, 21], while the other two types of 
nondegenerate companion result from systems with low-mass stars and thus should produce longer delay times.

Multidimensional hydrodynamics simulations of the different channels are difficult because of the large dynamic ranges required and the large binary parameter space to explore. Simulations following the ejecta evolution within the progenitor system starting from a pointlike explosion potentially make the most direct contact with supernova observations and progenitor searches, though they do not address the question of how the supernova explosion begins. Such simulations can determine the amount of mass that should be unbound from the companion, determine whether hydrogen lines should be seen, determine the level of asymmetry introduced in the supernova remnant by interaction with the companion, and provide clues regarding the appearance of the post-supernova companion.

Marietta et al. [22] explored the RG, subgiant, and MS single-degenerate channels using 2D Eulerian hydrodynamics simulations. They found that significant quantities of hydrogen should be unbound from the companion star envelope in each case ( $15 \%$ for MS and subgiant cases, and $98 \%$ for the RG), in conflict with observational upper limits $[6,7]$. They also found that the companion star blocks about $7-12 \%$ of the SN ejecta, introducing anisotropy in the supernova remnant and possibly having consequences for Si II spectral line shapes, but that the kick delivered to the companion is smaller than the orbital speed in each case and negligible for the RG core. Fallback of part of the MS or subgiant companion's envelope produces a dramatic increase in the companion's luminosity, but relatively little contamination by $\mathrm{SN}$ ejecta occurs. The remnant in the RG case is a helium pre-white dwarf with an extended hydrogen-rich envelope of very low mass (about $0.02 M_{\odot}$ ) that should appear as an underluminous $\mathrm{O} / \mathrm{B}$ star for up to $10^{6} \mathrm{yr}$.

More recently, Pakmor et al. [23] updated these results to consider the effect that pre-supernova binary evolution has on the structure of MS companions, as suggested by [24], using 3D smoothed particle hydrodynamics (SPH) simulations. Pakmor et al. used initial conditions derived from stellar models [9] that included binary mass transfer and thus yielded a more compact MS companion. Because of this, they found a tenfold reduction in the amount of unbound mass, bringing the predicted amount back into agreement with observational upper limits. They also found strong dependences of the unbound mass $M_{\text {unbound }}$ and kick velocity $v_{\text {kick }}$ on initial binary separation $a$, with $M_{\text {unbound }} \propto a^{-3.49}$ and $v_{\text {kick }} \propto a^{-1.45}$. Their use of SPH with a relatively small number of particles $\left(\sim 10^{6}\right)$ caused them to fail to reproduce the detailed fluid instabilities seen by Marietta et al., but they performed a convergence study using the same MS model as Marietta et al. and found good agreement for the unbound mass and kick velocity. These quantities therefore should not be too sensitive to the method used, though we note that the degree of contamination of the companion by SN ejecta should depend significantly on the treatment of small-scale instabilities.

We are revisiting this problem using modern adaptive mesh refinement (AMR) techniques, larger computers, and companion models that incorporate the effects of binary evolution. These advances permit us to consider larger 3D spatial dynamic ranges using the same type of Eulerian shock-capturing hydrodynamics methods as Marietta et al. We also examine the helium-star channel, which has not heretofore been considered using hydrodynamical simulations. We have previously reported on 2D simulations of the helium-star channel in [25]; here we summarize these results and present preliminary $3 \mathrm{D}$ results for the RG channel.

\section{NUMERICAL METHODS}

We use two separate codes to create progenitor stellar models and to evolve SN explosions within binary systems. To construct 1D stellar models including mass loss due to binary interactions, we use EZ [26], which is based on the STARS stellar evolution code originally developed by Eggleton [27, 28]. We have modified the code to include a mass loss term to account for binary mass transfer and wind losses. Using parameter values chosen from [9] (for MS and RG companions) or [21] (for helium-star companions), we evolve each companion star model up to the point at which the supernova explosion is expected to take place. We then interpolate the 1D profiles of density, temperature, and isotopic abundances onto the 2D or 3D grid used in the next stage of the calculations. We allow the multidimensional stellar model to relax for several dynamical times in order to reduce interpolation errors.

To simulate the explosion, we employ FLASH 3.2 [29, 30], a parallel Eulerian AMR hydrodynamical code based on the piecewise parabolic method or PPM [31] for hydrodynamics and a direct multigrid algorithm [32] for gravity. We use a nonideal equation of state (EOS) appropriate to stellar material that tabulates the Helmholtz free energy as a function of density, temperature, and composition [33]. We separately track hydrogen, helium, oxygen, and carbon outside the SN ejecta, while the ejecta themselves are assigned a pure nickel abundance.

FLASH uses the block-structured AMR package PARAMESH [34], which manages an oct-tree mesh that is distributed in parallel using a space-filling curve. To capture flow discontinuities, we refine blocks based on the second derivative of the gas density and pressure. We ignore the second-derivative criterion in blocks where the maximum density falls below a preset threshold $\left(10^{-6} \mathrm{~g} \mathrm{~cm}^{-3}\right)$ in order to avoid excessively refining low-density regions. To 
cope with features peculiar to each of our models, we employ additional refinement criteria as described below.

Even with AMR, degenerate RG cores are too small for us to resolve directly with our mesh while still achieving high resolution elsewhere in our computational domain. Therefore we replace the core gas in RG companions with a spherical cloud of about $2 \times 10^{5}$ particles with radius three times the smallest zone spacing. The gravitational force on and due to the particles is determined using the particle-mesh method [35], with an important modification: all of the particles in the cloud move rigidly together with the cloud's center of mass. This arrangement ensures that the mapping of particle densities onto the mesh and of gravitational forces onto the cloud's center of mass are free of Cartesian grid effects introduced by the use of a cloud-in-cell (CIC) mapping kernel. In hydrostatic equilibrium tests of a single RG star, this technique allows us to maintain gas velocities in the envelope less than about $1 \%$ of the sound speed for more than a dynamical time. To ensure maximum mesh resolution of the core, we force refinement of all mesh blocks that contain any particles in the cloud. These methods have proven successful in related simulations of common-envelope evolution [36]. The MS and helium star companions do not have degenerate cores, so we do not use particle clouds with them, but since their outer density profiles are much steeper, we force refinement near their surfaces.

After allowing the companion star model to relax on a multidimensional AMR mesh, we introduce an SN Ia by adding a small spherical cloud of high-energy gas. To reduce Cartesian mesh effects, we force the mesh to refine about the explosion by two extra levels beyond the rest of the computational volume within a spherical region of radius five times the initial radius of the explosion. At this level of refinement the initial explosion radius is about three zones across. For Chandrasekhar-mass explosions, we use the W7 model [37] to set the ejecta mass, kinetic energy, and thermal energy. The W7 model involves a central carbon deflagration that provides a good fit to observed SN Ia light curves and spectra. In this model the explosion energy is $1.233 \times 10^{51} \mathrm{erg}$, and the average radially-directed ejecta velocity is $8.527 \times 10^{3} \mathrm{~km} \mathrm{~s}^{-1}$. For our purposes, we assume the entire ejecta mass $\left(1.378 M_{\odot}\right)$ consists of ${ }^{56} \mathrm{Ni}$.

\section{RESULTS}

We have created a preliminary 3D simulation of the RG case (Figure 1) for comparison with [22]. The RG in this simulation had a $0.36 M_{\odot}$ core and a total mass of $1.05 M_{\odot}$. The initial binary separation was $6.2 \times 10^{12} \mathrm{~cm}$, and the minimum zone spacing was about $7.3 \times 10^{10} \mathrm{~cm}$ within a computational volume of size 5 AU $\left(7.5 \times 10^{13} \mathrm{~cm}\right)$. These initial conditions resulted in Roche-lobe overflow, so the system was followed for one orbit with the unexploded WD represented by a particle cloud (as with the RG core) to develop a realistic aspherical shape. To initiate the explosion, we removed the WD particle cloud and replaced it with a gas sphere as described in the previous section.

Figure 1 shows the double shock structure that forms in this simulation when the SN ejecta begin to interact with the red giant. The forward and reverse shocks are separated by a contact discontinuity. When the reverse shock reaches the companion, a bow shock structure forms. We reproduce many features of the RG companion case in [22], but in our simulation an emptier central region is left behind by the ejecta, because Marietta et al. imposed their explosion at the grid boundary, whereas ours is imposed inside the simulation box. Moreover, because we include the orbital motion in 3D, the ejecta become somewhat asymmetric in our calculation. We use the kinetic, thermal, and potential energy of the gas in each zone to determine the total amount of bound and unbound gas. In agreement with [22], we find that almost all the RG envelope is unbound. (Note that neither simulation included the effect of binary mass transfer on the initial companion model.) However, a small amount of hydrogen is still left around the RG core.

Our investigation of the helium-star channel [25] has been more thorough than for the RG case, but our simulations to date have been 2D and thus have not included orbital motion. We assumed axisymmetry and placed the supernova and the helium star on the symmetry axis. Because helium stars are much more compact than red giants, for these simulations we used box sizes of $1-5 \times 10^{11} \mathrm{~cm}$ and minimum zone spacings of $5 \times 10^{7} \mathrm{~cm}$ to $1.2 \times 10^{8} \mathrm{~cm}$.

Qualitatively, our results are similar to the results of [22] and [23] for the MS case, but with a more compact companion and smaller binary separation. We performed a parameter survey using four different helium-star companions suggested by [21], described in Table 1, and varying initial binary separations. The helium stars all have relatively low masses at the time of the supernova explosion, but they evolve from MS stars of masses $5-8 M_{\odot}$. The white dwarfs in these models are the remnants of MS stars with initial masses $2-6.5 M_{\odot}$ and have accreted material up to the Chandrasekhar mass in the course of the pre-supernova binary evolution.

Figure 2 shows the unbound mass (defined as total unbound helium) and the helium-star kick velocity (defined as the center-of-mass velocity of the bound helium) as functions of binary separation in our simulations. We include $14 \%$ error bars based on results of a convergence test. The unbound mass can be fit by the relation

$$
M_{\mathrm{unbound}}=C_{\mathrm{ub}} a^{m_{\mathrm{ub}}} M_{\odot},
$$



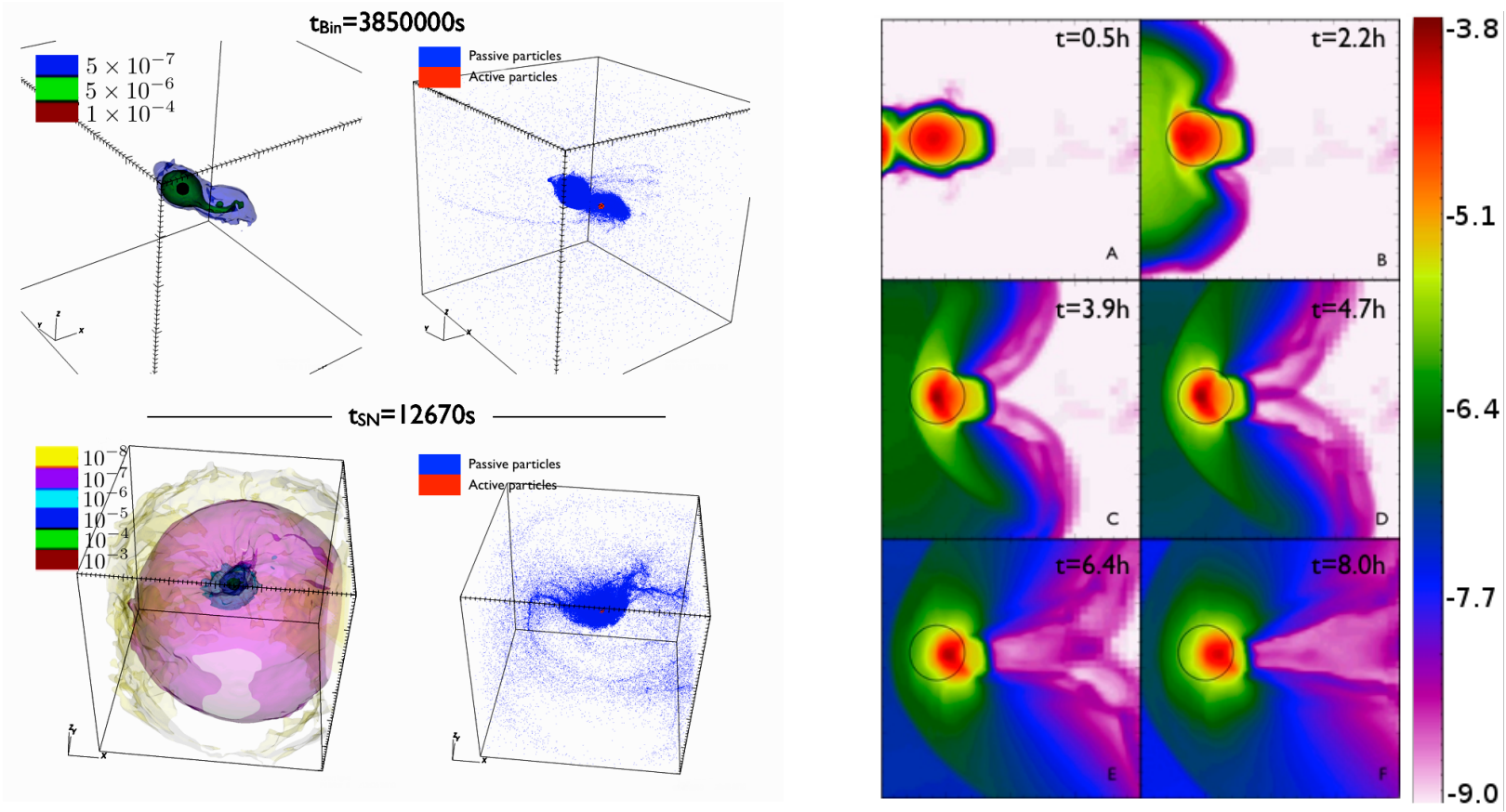

FIGURE 1. Left panel: Isodensity surfaces (left) and particle positions (right) for an RG-WD binary after $3.85 \times 10^{6} \mathrm{~s}$ of binary evolution (top) and 12,670 s after a supernova explosion (bottom). Passive tracer particles are blue, while active particles representing the WD companion are red. The box size is about $2 \mathrm{AU}\left(3 \times 10^{13} \mathrm{~cm}\right)$. Right panel: Density distribution in the orbital plane for RG model at different times after a supernova explosion. The circle shows the initial RG size and position. Each frame spans a distance of $1 \mathrm{AU}\left(1.5 \times 10^{13} \mathrm{~cm}\right)$. The color bar shows log density in $\mathrm{g} \mathrm{cm}^{-3}$.

where $a$ is the orbital separation, $m_{\mathrm{ub}}$ is the power-law index, and the constant $C_{\mathrm{ub}}$ depends only on the heliumstar model (see Table 1). For comparison, we also plot the power-law relation with index -3.49 found by [23] and the data from [22] for MS companions (consistent with an index of -3.14). The power-law indices for our heliumstar companions vary in a small range and bracket their results, suggesting that the index may be insensitive to the evolutionary state of the companion. The normalization of the above relation does appear to be sensitive to the nature of the companion star.

At early times, the helium star velocity varies dramatically, but after about 1000 seconds it settles down to a roughly constant value. We use an appropriate time average of this value as the kick velocity. For initial binary separations larger than 4 times the helium-star radius $R_{\mathrm{He}}$ the kick velocity could not be adequately determined because of the initial potential perturbation of the WD. As obtained by [23] and [24], a power-law relation is also found in our simulation and can be fitted by the relation

$$
v_{\text {kick }}=C_{\text {kick }} a^{m_{\text {kick }}},
$$

where $v_{\text {kick }}$ is the kick velocity, $m_{\text {kick }}$ is the power-law index, and the constant $C_{\text {kick }}$ depends only on the helium-star model (see Table 1). However, unlike the situation for the final unbound mass, the slope is very different from that

TABLE 1. Helium-star models considered in [25] with best-fit power-law slopes.

\begin{tabular}{ccccc}
\hline Model & $\begin{array}{c}\text { Mass } \\
\left(M_{\odot}\right)\end{array}$ & $\begin{array}{c}\text { Radius } \\
\left(10^{10} \mathrm{~cm}\right)\end{array}$ & $m_{\mathrm{ub}}$ & $m_{\text {kick }}$ \\
\hline He-WDa & 0.697 & 0.63 & -4.01 & -3.28 \\
He-WDb & 0.803 & 1.10 & -3.13 & -2.90 \\
He-WDc & 1.007 & 1.35 & -3.48 & -3.18 \\
He-WDd & 1.206 & 1.63 & -3.51 & -2.71 \\
\hline
\end{tabular}



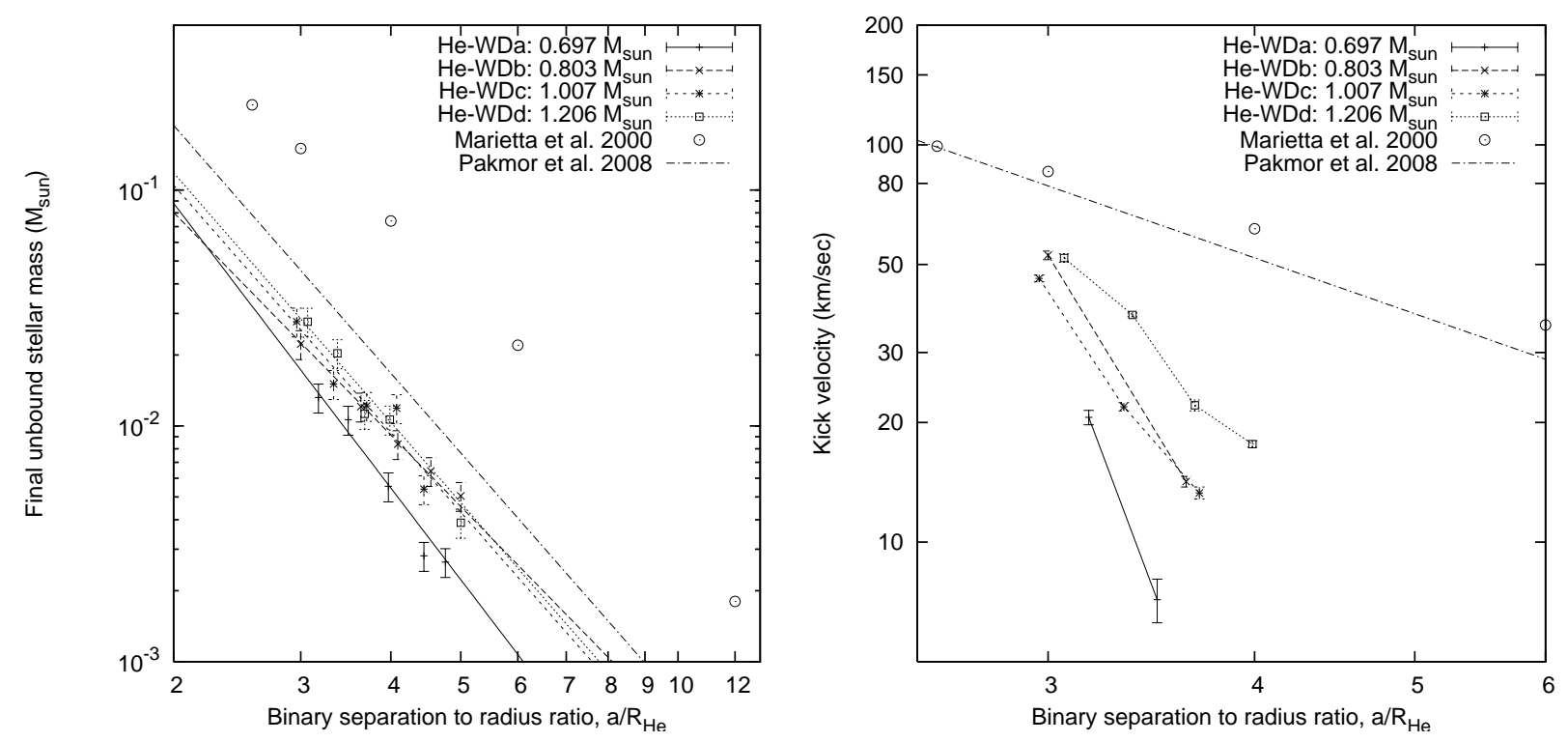

FIGURE 2. Unbound mass and kick velocity versus initial binary separation for our different $2 \mathrm{D}$ helium-star models. Separation is scaled to the helium-star radius $R_{\mathrm{He}}$.

found in the case of MS companions ( $m_{\text {kick }}=-1.45$ in [23], and $m_{\text {kick }}=-1.26$ in [22]). Based on a comparison with the analytical model of [24], we argue that this difference in slopes may arise because ablation (shock heating) and stripping (momentum transfer) have differing levels of importance in the helium-star case versus the RG and MS cases. The relative contributions of these processes to mass loss from the companion can be determined by examining the kinetic-to-thermal energy ratio of unbound helium as in [25] or, more accurately, by using passive tracer particles (Figure 1) and determining this energy ratio at the instant they become unbound.

The kick velocities for helium-star companions differ by a factor of 2 or more from RG and MS companions when considering separations characteristic of Roche-lobe overflow $\left(a_{\mathrm{RLOF}} \approx 3 R_{\mathrm{He}}\right.$ for the models considered here). However, for helium stars the final velocity of the companion is dominated by the pre-SN orbital velocity. Assuming the orbital velocity to be perpendicular to the kick, we find that the kick velocity is only about $20 \%$ of the resultant.

\section{DISCUSSION AND CONCLUSIONS}

While hydrogen-rich and helium-rich companion stars show many of the same qualitative effects when struck by supernova ejecta, the degree of compactness of the companion plays a very important role in determining how much mass is lost from the companion and how much of a kick it receives. It remains to be seen whether the ratio of ablated mass to stripped mass also depends on the degree of compactness, as this ratio has not yet been computed for the RG and MS cases. The companion's compactness increases when we consider stars that have already lost mass due to binary interactions. This suggests that an understanding of the outcomes of common-envelope evolution is crucial for identifying the types of companions most likely to encounter a single-degenerate SN Ia situation.

Our next steps are to revisit the RG and MS channels via 3D AMR simulations that include the effects of binary evolution and to examine the helium-star channel in 3D. 3D simulations give us better estimates of the amount of SN ejecta material that remains bound to the companion star, since the development of the instabilities that drive mixing depends on grid dimensionality. They should also permit us to determine more accurately the fraction of sightlines to the SN that can be obscured by the companion. Using tracer particles in 3D, we can also more accurately determine the relative amounts of stripped and ablated material (discussed using tracer fluids in [25]). Finally, by including radiation transport we expect to determine what fraction of the hydrogen and helium unbound by the supernova is ionized by radiation from the companion star. 


\section{ACKNOWLEDGMENTS}

The simulations presented here were carried out using the NSF Teragrid's Ranger system at the Texas Advanced Computing Center under allocation TG-AST040034N. FLASH was developed largely by the DOE-supported ASC/ Alliances Center for Astrophysical Thermonuclear Flashes at the University of Chicago. This work was supported, in part, by NSF AST-0703950 to Northwestern University. PMR gratefully acknowledges the International Travel Grant program of the American Astronomical Society.

\section{REFERENCES}

1. J. Whelan, and I. Iben, Astrophysical Journal 186, 1007-1014 (1973).

2. D. Homeier, D. Koester, H. Hagen, S. Jordan, U. Heber, D. Engels, D. Reimers, and S. Dreizler, Astronomy and Astrophysics 338, 563-575 (1998).

3. K. Nomoto, Astrophysical Journal 253, 798-810 (1982).

4. I. Iben, and A. V. Tutukov, Astrophysical Journal Supplement Series 54, 335-372 (1984).

5. R. F. Webbink, Astrophysical Journal 277, 355-360 (1984).

6. S. Mattila, P. Lundqvist, J. Sollerman, C. Kozma, E. Baron, C. Fransson, B. Leibundgut, and K. Nomoto, Astronomy and Astrophysics 443, 649-662 (2005).

7. D. C. Leonard, Astrophysical Journal 670, 1275-1282 (2007).

8. I. Hachisu, M. Kato, and K. Nomoto, Astrophysical Journal 470, L97 (1996).

9. N. Ivanova, and R. E. Taam, Astrophysical Journal 601, 1058-1066 (2004).

10. F. Mannucci, M. D. Valle, N. Panagia, E. Cappellaro, G. Cresci, R. Maiolino, A. Petrosian, and M. Turatto, Astronomy and Astrophysics 433, 807-814 (2005).

11. G. Nelemans, L. R. Yungelson, S. F. P. Zwart, and F. Verbunt, Astronomy and Astrophysics 365, $491-507$ (2001).

12. R. Napiwotzki, N. Christlieb, H. Drechsel, H. Hagen, U. Heber, D. Homeier, C. Karl, D. Koester, B. Leibundgut, T. R. Marsh, S. Moehler, G. Nelemans, E. Pauli, D. Reimers, A. Renzini, and L. Yungelson, Astronomische Nachrichten 322, 411-418 (2001).

13. R. Napiwotzki, D. Koester, G. Nelemans, L. Yungelson, N. Christlieb, A. Renzini, D. Reimers, H. Drechsel, and B. Leibundgut, Astronomy and Astrophysics 386, 957-963 (2002).

14. K. Nomoto, and I. Iben, Astrophysical Journal 297, 531-537 (1985).

15. L. Dessart, A. Burrows, C. D. Ott, E. Livne, S. Yoon, and N. Langer, Astrophysical Journal 644, 1063-1084 (2006).

16. D. T. Wickramasinghe, J. R. Hurley, L. Ferrario, C. A. Tout, and P. D. Kiel, Journal of Physics Conference Series 172, 2037 (2009).

17. L. R. Yungelson, and M. Livio, Astrophysical Journal 528, 108-117 (2000).

18. M. Kato, and I. Hachisu, Astrophysical Journal Letters 613, L129-L132 (2004).

19. F. Mannucci, M. D. Valle, and N. Panagia, Monthly Notices of the Royal Astronomical Society 370, 773-783 (2006).

20. É.. Aubourg, R. Tojeiro, R. Jimenez, A. Heavens, M. A. Strauss, and D. N. Spergel, Astronomy and Astrophysics 492, 631-636 (2008).

21. B. Wang, X. Meng, X. Chen, and Z. Han, Monthly Notices of the Royal Astronomical Society 395, 847-854 (2009).

22. E. Marietta, A. Burrows, and B. Fryxell, Astrophysical Journal Supplement Series 128, 615-650 (2000).

23. R. Pakmor, F. K. Röpke, A. Weiss, and W. Hillebrandt, Astronomy and Astrophysics 489, 943-951 (2008).

24. X. Meng, X. Chen, and Z. Han, Publications of the Astronomical Society of Japan 59, 835-840 (2007).

25. K. Pan, P. M. Ricker, and R. E. Taam, Astrophysical Journal 715, 78-85 (2010).

26. B. Paxton, Publications of the Astronomical Society of the Pacific 116, 699-701 (2004).

27. P. P. Eggleton, Monthly Notices of the Royal Astronomical Society 151, 351 (1971).

28. P. P. Eggleton, Monthly Notices of the Royal Astronomical Society 156, 361 (1972).

29. B. Fryxell, K. Olson, P. Ricker, F. X. Timmes, M. Zingale, D. Q. Lamb, P. MacNeice, R. Rosner, J. W. Truran, and H. Tufo, Astrophysical Journal Supplement Series 131, 273-334 (2000).

30. A. Dubey, K. Antypas, M. K. Ganapathy, L. B. Reid, K. Riley, D. Sheeler, A. Siegel, and K. Weide, Parallel Computing 35 , 512-522 (2009).

31. P. Colella, and P. R. Woodward, Journal of Computational Physics 54, 174-201 (1984).

32. P. M. Ricker, Astrophysical Journal Supplement Series 176, 293-300 (2008).

33. F. X. Timmes, and F. D. Swesty, Astrophysical Journal Supplement Series 126, 501-516 (2000).

34. P. MacNeice, K. M. Olson, C. Mobarry, R. de Fainchtein, and C. Packer, Computer Physics Communications 126, 330-354 (2000).

35. R. W. Hockney, and J. W. Eastwood, Computer Simulation Using Particles, A. Hilger, Bristol [England], 1988.

36. P. M. Ricker, and R. E. Taam, Astrophysical Journal 672, L41-L44 (2008).

37. K. Nomoto, F. Thielemann, and K. Yokoi, Astrophysical Journal 286, 644-658 (1984). 